\title{
Bioclipse 2: A scriptable integration platform for the life sciences
} Ola Spjuth*1, Jonathan Alvarsson'1, Arvid Berg1, Martin Eklund1, Stefan Kuhn², Carl Mäsak¹, Gilleain Torrance2, Johannes Wagener ${ }^{3}$, Egon L Willighagen ${ }^{1}$, Christoph Steinbeck ${ }^{2}$ and Jarl ES Wikberg ${ }^{1}$

\author{
Address: ${ }^{1}$ Department of Pharmaceutical Biosciences, Uppsala University, Uppsala, Sweden, ${ }^{2}$ Wellcome Trust Genome Campus, European \\ Bioinformatics Institute, Hinxton, UK and ${ }^{3}$ Max von Pettenkofer-Institut, Ludwig-Maximilians-Universität, Munich, Germany \\ Email: Ola Spjuth* - ola.spjuth@farmbio.uu.se; Jonathan Alvarsson - jonathan.alvarsson@farmbio.uu.se; \\ Arvid Berg - arvid.berg@farmbio.uu.se; Martin Eklund - martin.eklund@farmbio.uu.se; Stefan Kuhn - stefan.kuhn@ebi.ac.uk; \\ Carl Mäsak - carl.masak@farmbio.uu.se; Gilleain Torrance - maclean@ebi.ac.uk; Johannes Wagener - johannes.wagener@med.uni- \\ muenchen.de; Egon L Willighagen - egon.willighagen@farmbio.uu.se; Christoph Steinbeck - steinbeck@ebi.ac.uk; \\ Jarl ES Wikberg - jarl.wikberg@farmbio.uu.se \\ * Corresponding author
}

Published: 3 December 2009

BMC Bioinformatics 2009, 10:397 doi:10.1 186/147|-2105-10-397
Received: 21 August 2009

Accepted: 3 December 2009

This article is available from: http://www.biomedcentral.com/I47I-2/05//0/397

(C) 2009 Spjuth et al; licensee BioMed Central Ltd.

This is an Open Access article distributed under the terms of the Creative Commons Attribution License (http://creativecommons.org/licenses/by/2.0), which permits unrestricted use, distribution, and reproduction in any medium, provided the original work is properly cited.

\begin{abstract}
Background: Contemporary biological research integrates neighboring scientific domains to answer complex questions in fields such as systems biology and drug discovery. This calls for tools that are intuitive to use, yet flexible to adapt to new tasks.

Results: Bioclipse is a free, open source workbench with advanced features for the life sciences. Version 2.0 constitutes a complete rewrite of Bioclipse, and delivers a stable, scalable integration platform for developers and an intuitive workbench for end users. All functionality is available both from the graphical user interface and from a built-in novel domain-specific language, supporting the scientist in interdisciplinary research and reproducible analyses through advanced visualization of the inputs and the results. New components for Bioclipse 2 include a rewritten editor for chemical structures, a table for multiple molecules that supports gigabyte-sized files, as well as a graphical editor for sequences and alignments.
\end{abstract}

Conclusion: Bioclipse 2 is equipped with advanced tools required to carry out complex analysis in the fields of bio- and cheminformatics. Developed as a Rich Client based on Eclipse, Bioclipse 2 leverages on today's powerful desktop computers for providing a responsive user interface, but also takes full advantage of the Web and networked (Web/Cloud) services for more demanding calculations or retrieval of data. The fact that Bioclipse 2 is based on an advanced and widely used service platform ensures wide extensibility, making it easy to add new algorithms, visualizations, as well as scripting commands. The intuitive tools for end users and the extensible architecture make Bioclipse 2 ideal for interdisciplinary and integrative research.

Bioclipse 2 is released under the Eclipse Public License (EPL), a flexible open source license that allows additional plugins to be of any license. Bioclipse 2 is implemented in Java and supported on all major platforms; Source code and binaries are freely available at http://www.bioclipse.net. 


\section{Background}

Contemporary biological research integrates neighboring scientific domains to answer complex questions in fields such as systems biology and drug discovery [1]. To this end, researchers combine diverse types of data from increasingly available public sources. This calls for flexible analysis tools which can quickly be adapted to new and unforseen tasks through open interfaces and extensible mechanisms. Workflows have been proposed as one solution for this problem, and have been shown to perform well in several studies. While orchestration tools like Taverna [2] are well equipped for producing workflows with reproducible and reusable functionality, a workbench is focused on iterative science where analysis and visualization tools are available for data exploration. In order to meet the demands of today's data-intensive problem settings, a workbench must be able to automate operations on large scale and produce executive summaries of analyses.

The Bioclipse workbench [3], presented here in an improved version, constitutes such a flexible analysis framework. It is a free, open source workbench that allows users to work with resources and entities in the life sciences, such as chemical structures, sequences, spectra, and alignments. Following its first release in early 2007, Bioclipse has, as of September 2009, been downloaded more than 30000 times, and been awarded 3 international prizes for its innovative architecture. Bioclipse 2 constitutes a complete rewrite which provides the project with a strong foundation for integrating life science components, and turns Bioclipse into a stable, scalable platform for the life sciences. A major update from previous versions is that Bioclipse 2 is completely scriptable, allowing to scale up analyses by automating functionality in the Bioclipse Scripting Language (BSL) and also enables sharing of reproducible scripts.

\section{Implementation}

Bioclipse is built in Java on the Eclipse Rich Client Platform (RCP) http://www.eclipse.org/rcp which has a component-based architecture where all components (including the core ones) are referred to as plugins. The plugin architecture builds on the OSGi framework [4], which is a dynamic Java-based component model. There is an entire ecosystem of Eclipse plugins available in various domains such as mathematics, finance, education, and software development. While taking advantage of the existing plugins, Bioclipse extends this ecosystem with a domain object model and implementations for the life sciences. It is very easy to contribute new plugins for Bioclipse, and tutorials are available on the Bioclipse wiki. While the integration with the Bioclipse platform needs to be written in Java, the plugins' functionality can be implemented in any language. The use of a standardized plugin architecture is a major advantage over many existing plat- forms in the life sciences who provide their own implementations. In fact, prominent applications such as Taverna [2] and Cytoscape [5] have recently announced that future versions will be based on OSGi due to its many advantages.

In Bioclipse 2, all functional code contributed by plugins is structured in Bioclipse Managers; e.g. code that provides access to 3D conformer generation using Balloon [6] are available in the BalloonManager. Bioclipse 2 makes use of Spring http://www.springsource.org to provide dependency injection and aspect oriented programming (AOP), which allows for encapsulating concerns into separate entities. Bioclipse 2 makes us of this to inspect Manager calls and decide e.g. if calls should be run in separate threads, and also records method usage. Annotated Manager objects are published into the scripting environment, and hence the same objects that are called from the GUI are reachable from scripts.

The life sciences make extensive use of the Web as a medium for distributing data and software tools. As a Rich Client, Bioclipse takes full advantage of this service-oriented architecture (SOA), and provides a platform to integrate Web services using various technologies, including SOAP, REST, and XMPP + IO Data [7]. The latter is a novel technology which enables truly asynchronous communication between the client and server.

\section{Results and Discussions Cheminformatics}

Cheminformatics comprises the management, analysis, and visualization of chemical structures and related information. Bioclipse 2 includes a completely rewritten version of the 2D editor JChemPaint [8] with advanced chemical editing and visualization features, a new MoleculesTable that supports editing of resources containing multiple molecules (see Figure 1), and the latest version of Jmol http://www.jmol.org for interactive 3D visualization. A domain model and extensive list of cheminformatics functionality is provided by the Chemistry Development Kit (CDK) [9] and Chemical Markup Language (CML) $[10,11]$; this includes for example $2 \mathrm{D}$ and 3D coordinates generation, SMARTS searches, distance measurements, fingerprint calculations, substructure searches, and property calculations including InChI, SMILES, and QSAR descriptors. Balloon [6] is also bundled for 3D conformer generation. The PubChem eUtils are used to query and retrieve compounds into Bioclipse, and several clients for demanding cheminformatics analyses are integrated as XMPP cloud services.

\section{Bioinformatics}

Bioinformatics in Bioclipse 2 comprises primarily the management and analysis of sequences, proteins, and related information. In contrast to earlier versions, Bio- 


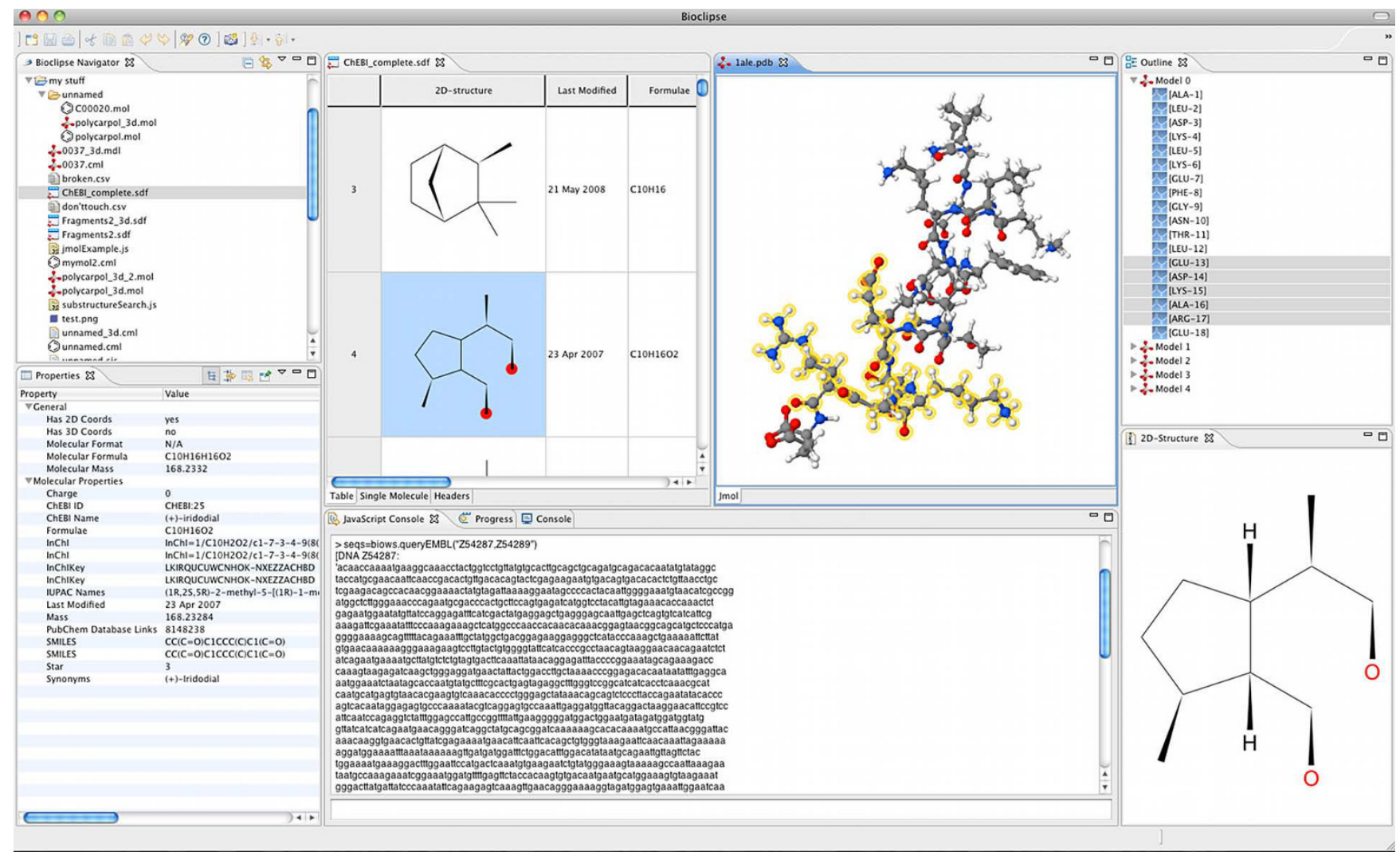

\section{Figure I}

Screenshot from Bioclipse 2. Screenshot from Bioclipse 2 showing the new Molecules Table (top middle panel) with chemical structures and properties in a spreadsheet-like editor and the selected structure rendered in a separate 2D view (bottom right panel), and computed properties (bottom left panel). Also shown is a 3D structure where monomers are selected in the outline (top rightmost panel) and highlighted in the interactive 3D visualization component Jmol (top right panel). The Javascript console (bottom middle panel) can be used to execute scripting commands.

clipse 2 features a Sequence Editor which allows for editing and visualization of sequences, including DNA, RNA, protein sequences, as well as pairwise and multiple alignments. The object model for sequence management in Bioclipse is primarily based on BioJava [12], which also provides common features like format conversions and translations. Examples of integrated Web services are Kalign for sequence alignment [13], and WSDbfetch for retrieving resources from public repositories at EBI [14].

\section{Spectral analysis}

The spectrum feature in Bioclipse was developed with three application scenarios in mind: An input facility for spectral data in the context of a computer-assisted structure elucidation tool $[15,16]$, the (offline) authoring of datasets for spectral databases [17], and as a tool for spectrum handling in the context of metabolomics. To these ends, a set of components were implemented which allow for parsing and editing of spectral data (NMR, MS) in open formats (JCAMP, CMLSpect), and visualization in interactive tables and graphs. Spectra can be assigned to chemical structures and this assignment can be displayed and browsed interactively by the user.

\section{Scripting integrative analysis}

The Bioclipse plugins can contribute functionality made available via an integrated scripting environment, for example Javascript. This can be seen as a domain-specific language (DSL) for the life sciences, and we have named this Bioclipse Scripting Language (BSL). In Bioclipse 2, BSL is based on Javascript, but we envision other implementations in the future. A Javascript Console (see Figure 1 ) is available for executing individual commands, and a Javascript editor can be used to set up and execute scripts (see Figure 2 for two sample scripts). The mix of plugincontributed methods with the JavaScript language has been shown to be very powerful, and makes it possible to seamlessly use integrated third party software in scripts that automate analyses.

Sharing of scripts is an important feature, as it allows for collaborators to reproduce analyses, and to provide the 
/ Download, filter, and generate 3D conformations list $=$ pubchem.download (pubchem.search $(" H I V "))$; pyrrolidine $=$ cdk.fromSMILES ("C1CNCC1"): matches = cdk.substructureMatches (list, pyrrolidine); ui.open (matches):

$\mathrm{c}=$ balloon $\cdot$ generate 3Dconformations (matches $\cdot \operatorname{get}(0), 5)$; cdk.saveSDFile("Virtual/conf.sdf", c);

(a)

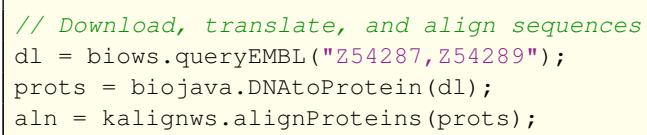

(b)

\section{Figure 2}

Example scripts for Bioclipse 2. The Bioclipse Scripting Language in Bioclipse 2 is based on Javascript with extensions contributed by plugins. Here are two example scripts that demonstrate several integrated components: a) PubChem is queried for compounds matching the name "HIV" using a Web service, substructures are isolated that contain pyrrolidine and opened for inspection, Balloon is used to generate 5 3D conformations of the first match, and finally the conformations are saved to an SDFile. b) Two nucleotide sequences are downloaded from EMBL using the WSDbfetch Web service at EBI, translated to protein using Biojava, and aligned using the Kalign Web service at EBI. The complete examples are available as gists from github with id: a) 163575 and b) 163440 .

means to extend Bioclipse with composite functionality for a specific task. Bioclipse supports sharing of scripts via the MyExperiment [18] and Gist [19] services. Dedicated plugins in Bioclipse makes it easy to download the latest version of a script using both services. Lists of scripts are available for MyExperiment on [20] and for Gist on del.icio.us on [21].

\section{Conclusion}

Bioclipse is an advanced integration platform for the life sciences featuring an easy-to-use workbench that delivers the latest functionality available from intuitive graphical editors and wizards, and enables users to take advantage of networked databases and online services. Experienced users will appreciate the scripting language for quickly executing powerful commands with the possibility to visualize results directly in $2 \mathrm{D}$ or $3 \mathrm{D}$, and the easy extension of Bioclipse with new functionality. The combination of seamlessly using the GUI and the scripting language is already an appreciated feature, for example dragging and dropping resources into the scripting console to simplify scripting. To the best of our knowledge there exists no other framework, open source of proprietary, which inte- grates cheminformatics with bioinformatics in an extensible scripting language.

Bioclipse has an active development community, see the Bioclipse wiki http://wiki.bioclipse.net for more information. Ongoing projects include chemical and biological databases, QSAR, predictive toxicology, metabolomics, semantic data fusion, and systems biology. The Bioclipse project honors the idea of the Blue Obelisk Community [22] and promotes an open development and welcomes new developers and contributors.

\section{Availability and requirements \\ Project name: Bioclipse}

\section{Project home page: http://www.bioclipse.net}

Operating system(s): Platform independent

Programming language: Java

License: Eclipse Public License (EPL)

Restrictions to use by non-academics: None

\section{Authors' contributions}

All authors are developers or administrators of the Bioclipse project and read and approved the final manuscript.

\section{Acknowledgements}

The authors would like to acknowledge all who has provided code, bug reports, and suggestions in the development of Bioclipse 2. This work was supported by the Swedish VR (04X-05957) and Uppsala University (KoF 07).

\section{References}

I. Ohlstein EH, Johnson AG, Elliott JD, Romanic AM: New strategies in drug discovery. Methods Mol Biol 2006, 3 I 6: I- II.

2. Oinn T, Addis M, Ferris J, Marvin D, Senger M, Greenwood M, Carver T, Glover K, Pocock MR, Wipat A, Li P: Taverna: a tool for the composition and enactment of bioinformatics workflows. Bioinformatics 2004, 20( I 7):3045-3054.

3. Spjuth $\mathrm{O}$, Helmus T, Willighagen EL, Kuhn S, Eklund M, Wagener J Murray-Rust P, Steinbeck C, Wikberg JES: Bioclipse: an open source workbench for chemo- and bioinformatics. BMC Bioinformatics 2007, 8:59.

4. OSGi Alliance [http://www.osgi.org]

5. Shannon P, Markiel A, Ozier O, Baliga NS, Wang JT, Ramage D, Amin $\mathrm{N}$, Schwikowski B, Ideker T: Cytoscape: a software environment for integrated models of biomolecular interaction networks. Genome Res 2003, I3( I I):2498-2504.

6. Vainio MJ, Johnson MS: Generating conformer ensembles using a multiobjective genetic algorithm. J Chem Inf Model 2007, 47(6):2462-2474.

7. Wagener J, Spjuth O, Willighagen EL, Wikberg JES: XMPP for cloud computing in bioinformatics supporting discovery and invocation of asynchronous web services. BMC Bioinformatics 2009, 10:279.

8. Krause S, Willighagen E, Steinbeck C: JChemPaint - Using the Collaborative Forces of the Internet to Develop a Free Editor for 2D Chemical Structures. Molecules 2000, 5:93-98. 
9. Steinbeck C, Hoppe C, Kuhn S, Floris M, Guha R, Willighagen EL: Recent developments of the Chemistry Development Kit (CDK) - an open-source Java library for chemo- and bioinformatics. Curr Pharm Des 2006, I 2(I7):2 I I |-2 I 20.

10. Murray-Rust P, Rzepa HS: Chemical markup, $\mathbf{X M L}$ and the World-Wide Web. 2. Information objects and the CMLDOM. J Chem Inf Comput Sci 200 I, 4 I (5): I I I3-I I 23.

11. Willighagen E: Processing CML Conventions in Java. Internet Journal of Chemistry 200I, 4:4

12. Holland RCG, Down TA, Pocock M, Prlic A, Huen D, James K, Foisy S, Drager A, Yates A, Heuer M, Schreiber MJ: BioJava: an opensource framework for bioinformatics. Bioinformatics 2008, 24(18):2096-2097.

13. Lassmann T, Sonnhammer ELL: Kalign-an accurate and fast multiple sequence alignment algorithm. BMC Bioinformatics 2005, 6:298.

14. Labarga A, Valentin F, Anderson M, Lopez R: Web services at the European Bioinformatics Institute. Nucleic Acids Res 2007:W6-II.

15. Steinbeck C: SENECA: A platform-independent, distributed, and parallel system for computer-assisted structure elucidation in organic chemistry. J Chem Inf Comput Sci 200I, $4 I(6): \mid 500-1507$

16. Han Y, Steinbeck C: Evolutionary-algorithm-based strategy for computer-assisted structure elucidation. J Chem Inf Comput Sci 2004, 44(2):489-498.

17. Steinbeck C, Krause S, Kuhn S: NMRShiftDB-constructing a free chemical information system with open-source components. J Chem Inf Comput Sci 2003, 43(6): 1733-1739.

18. De Roure D, Goble C, Stevens R: The design and realisation of the myExperiment Virtual Research Environment for social sharing of workflows. Future Generation Computer Systems 2009, 25(556 |-567 [http://dx.doi.org/10.1016/j.future.2008.06.010].

19. Gist [http://gist.github.com]

20. MyExperiment list for kind Bioclipse Scripting Language [http://www.myexperiment.org/search?type=work flows\&query=kind:(Bioclipse\%20Scripting\%20Language)]

21. Bioclipse Gists tagged on delicious [http://delicious.com/tag/ bioclipse+gist]

22. Guha R, Howard MT, Hutchison GR, Murray-Rust P, Rzepa H, Steinbeck C, Wegner J, Willighagen EL: The Blue Obelisk-interoperability in chemical informatics. I Chem Inf Model 2006, 46(3):99|-998.

Publish with Bio Med Central and every scientist can read your work free of charge

"BioMed Central will be the most significant development for disseminating the results of biomedical research in our lifetime. "

Sir Paul Nurse, Cancer Research UK

Your research papers will be:

- available free of charge to the entire biomedical community

- peer reviewed and published immediately upon acceptance

- cited in PubMed and archived on PubMed Central

- yours - you keep the copyright
BioMedcentral 